\title{
Iris Biometrics Recognition System and Comparison of Biometrics Techniques
}

\author{
Zohaib Bukhari \\ Kohat University of Technology \\ Pakistan
}

\author{
Bilal Shams \\ Kohat University of Technology \\ Pakistan
}

\begin{abstract}
This paper is totally based on iris recognition and biometrics comparison techniques. Author revealed the story of iris recognition and biometrics comparison and provided the step by step detail about iris biometrics recognition and also elaborated the use of iris recognition and mentioned the key role played by it in daily life.
\end{abstract}

\section{Keywords}

Iris recognition, Biometrics, Comparison

\section{INTRODUCTION}

BIometris is the most secure and practical verification tool. It can be obtained, thieved, or neglected and developing one is difficult. Biometrics evaluates person's unique physical or behavior features to identify or verify their distinctiveness [1].

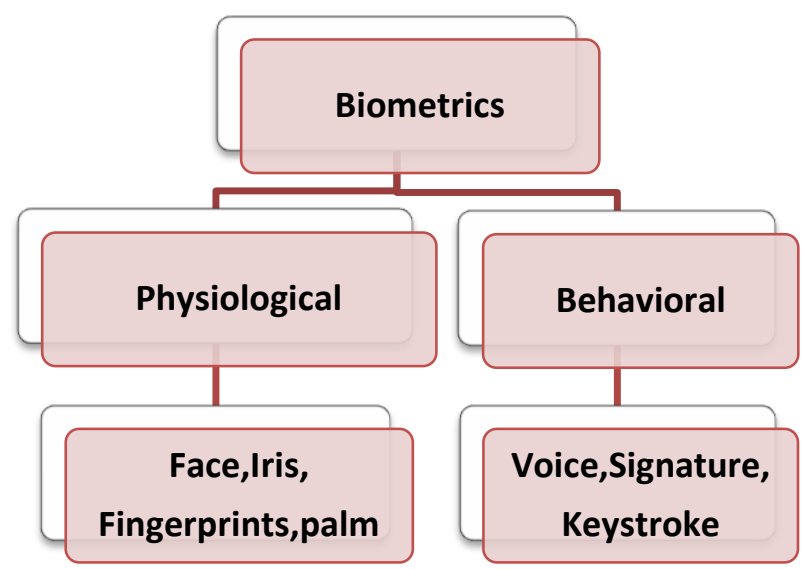

Figure 1.Types of Biometrics

Biometric identification system has developed for automatic verification and authentication, for example, finger recognition, face recognition, foot recognition, signature recognition, typing recognition, voice recognition, hand geometry. Biometric application mostly used for the purpose of commercial, government, e-heath, and for insecure area, for example. Atm, credit card, cellar phone, distance learning, ID card, driver's licenses, social security, passport control, terrorist identification ,missing children [2].

\section{IRIS RECOGNITION HISTORY}

Famous ophthalmologist Frank Buch in 1936 suggested the concept about iris using method's patterns to identify the individual
[13]. Dr Leonard and Aran ophthalmologist conceptualized that the two different irises are not a like they are different and in 1987 they also awarded regarding these differentiation concepts in iris method's pattern

Dr Flom collaborated with Dr John on the idea of automate generating method of human Iris .Nuclear agency supported the Idea and started to work and test the prototype unit in 1993, which was completed in 1995 very coordinately and successfully with the joint collaboration of two Drs. Dr Flom ,Daughman and safir in 1994. Dr Daughman awarded on this new idea of identification of human iris algorithms in 1995, and the patent idea of product first launched in commercial market in 2005 [14] and also provided the patent idea in globe market or other's competitors to develop their own idea of Iris recognition. The Iris patent code only introduced by Dr Daughman which will exist still 2011 [15].

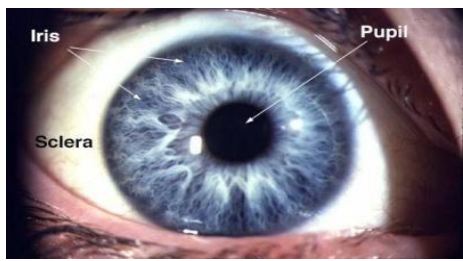

Figure 2.Iris diagram [3]

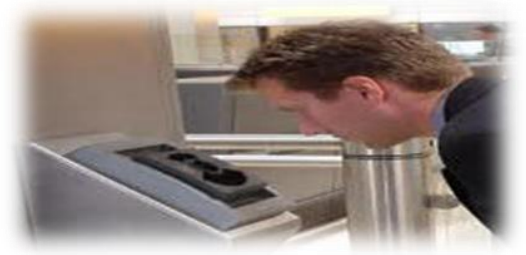

Figure 3.iris recognition system

\section{COMPARISON OF VARIOUS BIOMETRICS}

Various biometric aspects exist and are being used in different applications. Each biometric has its qualities and shortcomings, and the decision relies on the provision. No single biometric is relied upon to adequately meet the necessities of every last one of requisitions. In different statements, no biometric is "optimal." The match between a particular biometric and a requisition is dead set hinging on the operational mode of the provision and the lands of the biometric trademark. A concise prologue to the generally utilized biometrics is given beneath.

DNA: Deoxyribonucleic acid (DNA) is the one-dimensional (1-d) extreme interesting code for one's individual. It aside from the way 
that indistinguishable twins have indistinguishable DNA designs. It is, then again, at present utilized chiefly within the setting of scientific requisitions for individual recognition. Three issues breaking point the utility of this biometrics for different requisitions [2]: 1) defilement and affectability: it is simple to take a bit of DNA from a clueless subject that could be accordingly misused for a ulterior reason [9]; 2) programmed continuous recognition issues: the present engineering for DNA matching needs awkward concoction systems (wet forms) including a master's abilities and is not designed for on-line noninvasive recognition [9]; and 3) security issues: qualified data about susceptibilities of an individual to certain maladies could be picked up from the DNA example and there is a worry that the unintended misuse of hereditary code informative content might bring about separation, e.g., in employing practices[2].

Hand and finger geometry: Hand geometry recognition frameworks are dependent upon various estimations taken from the human hand, incorporating its shape, size of palm, furthermore lengths and widths of the fingers. Business hand geometry-based verification frameworks have been commissioned in countless areas far and wide. The procedure is extremely basic, moderately simple to utilize, and cheap. Ecological components, for example dry climate or single person aberrances, for example dry skin don't seem to have any negative consequences for the verification exactness of hand geometry-based frameworks. The geometry of the hand is most certainly not known to be extremely dissimilar and hand geometry-based recognition frameworks can't be scaled up for frameworks needing recognizable proof of a single person from an impressive populace. Further, hand geometry qualified data may not be invariant throughout the development time of youngsters. Furthermore, a single's adornments (e.g., rings) or impediments in mastery (e.g., from joint pain), might posture further tests in concentrating the right hand geometry qualified data. The physical size of a hand geometry-based framework is substantial, and it can't be installed in certain units such as laptops. There are verification frameworks accessible that are in view of estimations of just a couple of fingers (regularly, record and center) as a substitute for the whole hand. These de- indecencies are littler than those utilized for hand geometry, however still much bigger than those utilized as a part of some different biometrics (e.g., unique finger impression, confront, voice).

Gait: This is one of the more current advances and is yet to be scrutinized in additional detail. Essentially, gait is the particular way one strolls, and it is an intricate spatiotemporal biometrics. It shouldn't be extremely notable yet might be utilized as a part of some level security provisions. Gait is a behavioral biometric and may not continue as before over a long time of time, because of progress in figure weight or genuine cerebrum harm. Securing of step is comparable to obtaining a facial picture and may be an adequate biometric. Since motion picture arrangement is utilized to measure several distinctive developments this technique is computationally unmanageable.

Keystroke: It is accepted that every individual sorts on a console in a trademark manner. This is likewise not exceptionally dissimilar however it offers sufficient prejudicial qualified data to allow character verification. Keystroke elements is a behavioral biometric; for a few people, one could need to watch great varieties in run of the mill sorting examples. Point of interest of this strategy is that keystrokes of an individual utilizing a framework could be screened unpretentiously as that individual is keying informative data. A different issue to thick about here is security.

Fingerprint: A fingerprint is a pattern of ridges and furrows located on the tip of each finger. Fingerprints were utilized for individual recognizable proof for numerous hundreds of years and the matching correctness was extremely towering [7]. Designs have been concentrated by making an inked impression of the fingertip on paper. Today, minimized sensors furnish advanced pictures of these patterns.

Fingerprint recognition for recognizable proof gets the introductory picture through no nonsense output of the finger by immediate contact with a spectator apparatus that can likewise check for approving properties for example temperature and beat. Since the finger truly touches the checking gadget, the surface can come to be slick and overcast after rehashed utilize and decrease the affectability and dependability of optical scanners. Strong state sensors defeat this and other specialized troubles on the grounds that the covered silicon chip itself is the sensor. Strong state gadgets use electrical capacitance to sense the edges of the unique finger impression and make a smaller computerized picture. Today, an unique mark scanner expenses in the vicinity of 20 USD and has gotten competitive in countless (Portable computer). Progressively verification frameworks, pictures procured by sensors are utilized by the characteristic extraction module to register the characteristic qualities. The characteristic qualities normally compare to the position and introduction of certain basic focuses regarded as details focuses [8]. The matching procedure includes looking at the two-dimensional details designs extricated from the client's print with those in the template. One issue with the present unique finger impression recognition frameworks is that they require a great measure of computational assets.

Ear: Ear biometrics is acknowledged to be an improving innovation and not a considerable measure of work has been finished in this field in connection with other biometric routines. In spite of this, it is recognized that "the state of the ear and the structure of the cartilaginous tissue of the pinna are dissimilar. The ear recognition methodologies are dependent upon matching the separation of remarkable focuses on the pinna from a milestone area on the ear" [2]. Cummings et al. has uncovered a technique for ear biometric matching which recognizes a single $99.6 \%$ of the time [6]. This is not an exceptionally heightened rate acknowledging that it might neglect to recognize 40 out of 1000 individuals, which demonstrates that this innovation's infrastructure is still in its outset.

Facial Recognition: One of the front side biometrics fundamental points of interest is that this system is without hands and non-rude (Id can persistently be performed from a separation), which is remarkably endorsed by the larger part of clients. Both, static (e.g. "mug-shot") and alterable (e.g. airport) provisions focus on examination of the minimum susceptible to modifications (beard, additional weight, maturing and so forth.) facial range. It could be described as the triangle district between temples and lips [2] [7]. Just 15-20 of total 80 human face nodal focuses (crests and valleys of unique characteristics) are enough for workstation delicate ware to complete recognition proof [7]. Jain et al. discuss two generally mainstream recognition approaches [2]:

- $\quad$ Measuring the area and state of facial characteristics (e.g. separates between students or from nose to lip or jaw); 
- Investigating the for the most part face picture as " a Weighted combination the interest of number of authoritative faces" [2].

Some elements that can influence face biometrics correctness are:

- Level of illumination throughout catch (a few system require certain foundation or extraordinary lighting);

- Picture determination;

- Vicinity of contact lenses or glasses;

- Modification of the identifier with time (maturing);

- If picture database surpasses the measure of 10000 templates [7] (information space issue);

Security issue is additionally one of the perils (e.g. the point when a

\begin{tabular}{|c|c|c|c|c|c|c|c|}
\hline Biometric identifier & 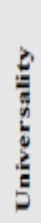 & 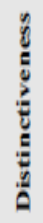 & 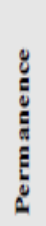 & 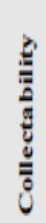 & $\frac{\mathscr{U}}{\frac{U}{\pi}}$ & 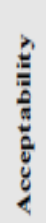 & ل: \\
\hline DNA & $\mathrm{H}$ & $\mathrm{H}$ & $\mathrm{H}$ & L & $\mathrm{H}$ & $\mathrm{L}$ & L \\
\hline Ear & $\mathrm{M}$ & M & $\mathrm{H}$ & $\mathrm{M}$ & $M$ & $\mathrm{H}$ & $M$ \\
\hline Face & $\mathrm{H}$ & L & $\mathrm{M}$ & $\mathrm{H}$ & L & $\mathrm{H}$ & $\mathrm{H}$ \\
\hline Facial thermogram & $\mathrm{H}$ & $\mathrm{H}$ & $\mathrm{L}$ & $\mathrm{H}$ & $M$ & $\mathrm{H}$ & L \\
\hline Fingerprint & $\mathrm{M}$ & $\mathrm{H}$ & $\mathrm{H}$ & $\mathrm{M}$ & $\mathrm{H}$ & $\mathrm{M}$ & $\mathrm{M}$ \\
\hline Gait & $\mathrm{M}$ & L & L & $\mathrm{H}$ & L & $\mathrm{H}$ & $\mathrm{M}$ \\
\hline Hand geometry & $\mathrm{M}$ & M & $\mathrm{M}$ & $\mathrm{H}$ & $\mathrm{M}$ & $\mathrm{M}$ & $\mathrm{M}$ \\
\hline Hand vein & $\mathrm{M}$ & M & $\mathrm{M}$ & $\mathrm{M}$ & M & $\mathrm{M}$ & L \\
\hline Iris & $\mathrm{H}$ & $\mathrm{H}$ & $\mathrm{H}$ & $\mathrm{M}$ & $\mathrm{H}$ & L & L \\
\hline Keystroke & L & L & L & $\mathrm{M}$ & L & $\mathrm{M}$ & $\mathrm{M}$ \\
\hline Odor & $\mathrm{H}$ & $\mathrm{H}$ & $\mathrm{H}$ & L & L & M & L \\
\hline Palmprint & $\mathrm{M}$ & $\mathrm{H}$ & $\mathrm{H}$ & $\mathrm{M}$ & $\mathrm{H}$ & $\mathrm{M}$ & $\mathrm{M}$ \\
\hline Retina & $\mathrm{H}$ & $\mathrm{H}$ & $\mathrm{M}$ & $\mathrm{L}$ & $\mathrm{H}$ & L & L \\
\hline Signature & $\mathrm{L}$ & $\mathrm{L}$ & $\mathrm{L}$ & $\mathrm{H}$ & $\mathrm{L}$ & $\mathrm{H}$ & $\mathrm{H}$ \\
\hline Voice & M & L & L & $\mathrm{M}$ & L & $\mathrm{H}$ & $\mathrm{H}$ \\
\hline
\end{tabular}

watched individual is not attentive to the reconnaissance). Jain et al. inquiry " if the front side itself, without any logical qualified data, is a sufficient premise for recognition an individual from a large number of characters with an amazingly elevated amount of certainty" [2].

Figure 4: Biometric Identifier compares' on by criteria High H; M - Medium; L - Low), [2]

i Universality: This application is general in its nature and hence, should be used with uniformity and equality.

ii Distinctiveness: The given trait must not be a sample of plagiarism and should be different in every aspect from the others.

iii Permanence: the biometric characteristic of an individual ought to be sufficiently invariant over a time of time as for the matching algorithm. (how changeless is the identifier?) iv Measurability: the biometric characteristic ought to be conceivable to gain, digitize and amiable to handling to concentrate illustrative characteristic sets, utilizing suitable mechanisms that don't reason hinder to the client.

v Performance: the recognition correctness and the assets needed to realize that precision might as well meet the obligations infringed by the biometric system.

vi Acceptability: people in the target populace that will use the biometric system ought to be eager to display their biometric characteristic to the system.(do individuals acknowledge it?)

vii Circumvention: hearty against false ambush.

A concise observation of the above biometric procedures dependent upon seven elements is given in Table 4. The relevance of a particular biometric system depends vigorously on the prerequisites of the provision realm. No single strategy can beat all the others in all operational situations. In this sense, every biometric system is acceptable and there is no optimal biometric trademark[2]. For instance, it is well realized that both the fingerprint-based and irisbased strategies are more exact than the voice-based method. On the other hand, in a tele-banking money provision, the voice-based procedure may be inclined toward since it might be joined seamlessly into the existing phone system [2].

\begin{tabular}{|l|l|}
\hline Biometrics & Crossover Accuracy \\
\hline Retinal Scan & $1: 10,000,000+$ \\
\hline Iris Scan & $1: 131,000$ \\
\hline Fingerprints & $1: 500$ \\
\hline Hand Geometry & $1: 500$ \\
\hline Signature Dynamics & $1: 50$ \\
\hline Voice Dynamics & $1: 50$ \\
\hline
\end{tabular}

Figure 5(b): Biometrics Comparison List [11]

\begin{tabular}{|l|l|l|l|l|}
\hline Method & $\begin{array}{l}\text { Code } \\
\text { Pattern }\end{array}$ & Misidentify & Security & Application \\
\hline $\begin{array}{l}\text { Iris } \\
\text { Recognition }\end{array}$ & Iris pattern & $1 / 120000$ & High & $\begin{array}{l}\text { High } \\
\text { Security } \\
\text { Facilities }\end{array}$ \\
\hline $\begin{array}{l}\text { Finger } \\
\text { printing }\end{array}$ & Fingerprints & $1 / 1000$ & Medium & Universal \\
\hline $\begin{array}{l}\text { Hand Shape } \\
\text { Size, }\end{array}$ & $\begin{array}{l}\text { Length } \\
\text { and } \\
\text { thickness }\end{array}$ & $1 / 700$ & Low & $\begin{array}{l}\text { Low- } \\
\text { security } \\
\text { facilities }\end{array}$ \\
\hline $\begin{array}{l}\text { Facial } \\
\text { Recognition }\end{array}$ & $\begin{array}{l}\text { Outline, } \\
\text { Shape and } \\
\text { distribution }\end{array}$ & $1 / 100$ & Low & $\begin{array}{l}\text { Low- } \\
\text { security } \\
\text { facilities }\end{array}$ \\
\hline Signature & $\begin{array}{l}\text { Shape } \\
\text { Of } \\
\text { Letters }\end{array}$ & $1 / 100$ & Low & $\begin{array}{l}\text { Low- } \\
\text { security } \\
\text { facilities }\end{array}$ \\
\hline $\begin{array}{l}\text { Voice } \\
\text { Printing }\end{array}$ & $\begin{array}{l}\text { Voice } \\
\text { characteri- } \\
\text { stic }\end{array}$ & $1 / 30$ & Low & $\begin{array}{l}\text { Telephone } \\
\text { service }\end{array}$ \\
\hline
\end{tabular}


Figure 5(a): Biometrics Comparison List [11]

\section{IRIS BIOMETRICS RECOGNITION STEPS}

Iris recognition has these following steps. Which perform basic works in iris biometrics recognition the following steps is.

1. Iris capture

2. Iris Localization

3. Iris Segmentation

4. Noise Detection

5. Normalization

6. Enhancement

7. Feature Extraction

8. Iris template, Matching

\subsection{Iris capture}

Actual iris identification can be broken down into four fundamental steps [16]. First, a person stands in front of the iris identification system, usually between one and three feet away, while a wide angle camera calculates the position of their eye. A second camera zooms in on the eye and takes a dark and white picture. After the iris system has one's iris in focus, it overlays a circular grid (zone's of analysis) on the picture of the iris and recognizes where areas of light and dim fall [16]. The purpose of overlaying the grid is so that the iris system can recognize a pattern within the iris and to generate 'points' within the pattern into an 'eye print' [16] At long last, the caught picture or 'eye print' is checked against a long ago archived 'reference template' in the database. The time it takes for an iris system to identify your iris is approximately two seconds [16].

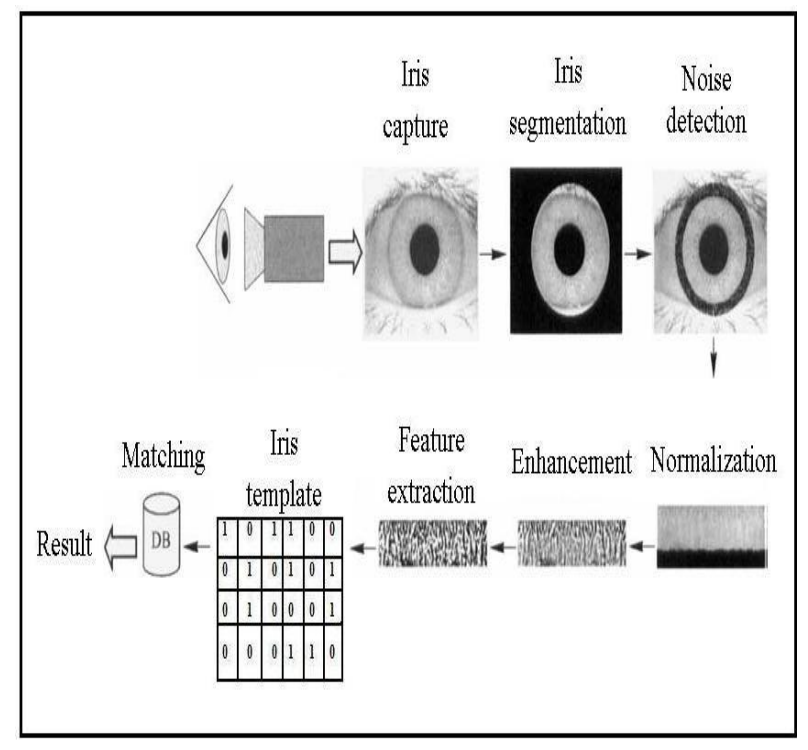

Figure 6.Iris biometrics recognition steps [5]

\subsection{Iris localization:}

An eye picture holds not just the iris area and yet a few parts that need to be differentiated from iris, for example the pupil, eyelids, sclera [10]. Consequently, at the first stage, segmentation ought to be finished to confine and separate the iris area from the eye picture. Iris localization is the detection of the iris range between pupil and sclera[10]. So we need to discover the upper and lower borders of the iris and confirm its inward and external circles. Two distinctive algorithms for localization of iris patterns are introduced here. The main algorithms is called dark rectangle algorithm that uses grey values for segmentation, second algorithms utilization Otsu thresholding and Hough round search for papillary boundary detection and slope scan strategy for limbic boundary detection[10]. Various algorithms has been created for iris localization. A large portion of them are dependent upon the Hough transform (Wields, 1997; Masek,2003)[10].

\subsection{Iris segmentation}

The process of partitioning a digital image into multiple segments is image segmentation. The main uses of Image segmentation are to locate objects and boundaries (lines, curves, etc.) in images. The process behind image segmentation is to assign a label to every pixel in an image such that pixels with the same label share certain visual characteristics. Segmentation techniques can be stated as the methods that are used for extracting and representing the information from an image [6].

\subsection{Noise Detection}

Eyelid curvature model is statistically created to evacuate the noisy points. In prior work they have proposed a technique dependent upon a 1-D rank channel to handle the eyelashes [19]. The eyelashes are mostly vertical thin and dim lines, and in this way could be debilitated or even killed by a 1-D level rank channel [19]. After rank filtering, edge detection is performed on the result iris picture along vertical heading [19]. One and only edge point is saved in every section so that most noisy edge points might be disregarded. Accordingly, a raw eyelid edge map Eraw is gotten [18]

\subsection{Normalization}

The irises captured from the distinctive people have different sizes. The measure of the irises from the same eye might change because of enlightenment varieties, separate from the camera, or different components. In the meantime, the iris and the pupil are non concentric [10]. When the iris area is portioned, the following stage is to normalize this part in order to empower the era of the iris code and their comparisons. Since the varieties in the eye, like optical size of the iris, position of pupil in the iris, and the iris introduction change from individual to individual, it is obliged to normalize the iris picture with the goal that the representation is normal to all, with comparative sizes [12].

\subsection{Enhancement}

Some cause of the acquiring device itself renders iris images unevenly illuminated [17]; this can be treated normally through histogram equalization. The interference of various noises is also present in the acquisition process; noise interference due to, for example, reflection of light is usually removed through homomorphic filtering [17]. If the acquired image for iris recognition is dim and unclear, the recognition capability of the iris recognition system will be greatly affected; a reconstruction based super-resolution method is usually used to improve the iris image. 
In a word, image enhancement is intended to reduce the effect of factors like uneven illumination and various noises on the recognition capability of an iris recognition system [17].

\subsection{Feature Extraction}

The most important step in automatic iris recognition is the capability of concentrating some exceptional characteristics from iris, which serve to create a particular code for every single person. Gabor and wavelet changes are normally utilized for analyzing the human iris designs and extracting features from them.

\subsection{Iris template, Matching}

It is extremely essential to show the acquired vector in a binary code because it is easier to determine the difference between two binary code-words than between two number vectors[12]. Actually, Boolean vectors are always easier to contrast and with control. We have applied a Hamming Distance matching calculation for the recognition of two simples [12]. It is essentially an exclusive Or (XOR) function between two bit patterns. Hamming Distance is a measure, which portray the contrasts, of iris codes. Every bit of displayed iris code is compared to the every bit of referenced iris code, if the two bits are the same, e.g. two 1's or two 0's, the system allocates a value " 0 " to that comparison and if the two bits are distinctive, the system allots a value "1" to that comparison [12]. Having localized the region of an acquired picture that compares to the iris, the final task is to choose if this pattern matches a formerly saved iris design. This matter of pattern matching could be decayed into four parts [4]

i Bringing the recently gained iris design into spatial alignment with a competitor data base entry;

ii Choosing a representation of the aligned iris patterns that makes their different patterns clear;

iii Evaluating the goodness of match between the recently gained and data base representations;

iv Deciding if the recently gained data and the data base entry were derived from the same iris dependent upon the goodness of match [4].

\section{CONCLUSION}

This paper gave a short introduction to the general concept of various biometrics, performing the comparison analysis of widely used biometric identifiers and their recognition techniques.

\section{FUTURE WORK}

The joined together special Gabor filters with wavelet maxima segments provide more textured informative data, since wavelet maxima permit us to effectively identify the horizontal and vertical parts through scale's variety and when the proposed special Gabor filters are connected on these segments at distinctive introductions and scales, more and exact informative data can been acquired consequently enhancing the exhibition of the recognition correctness.

The moment invariants are helpful and are effective to stand for iris emphasizes since they are touchy to interpretation, scaling and rotation and accordingly they give a complete, compact and significant feature vector which can enhance the matching stage while making it faster.

\section{REFERENCES}

[1] http://www.biometricsinstitute.org/pages/types-of-biometrics .html

[2] Anil K. Poor, Arun Ross and Salil Prabhakar(2004)"An introduction to Biometrics Recognition".

[3] http://www.peterindia.net/BiometricsView .html.

[4] Richard p.wildes, "An Emerging Biometrics Technology" Proceeding of the IEEE VOL.85,NO,9, September 1997.

[5] “UTM" http://se.fsksm.utm.my/iriscrypto/

[6] A Survey Level Set Method for Image Segmentation: (Jan. Feb. 2013)

[7] D. Maio, D. Maltoni, R. Cappelli, J. L. Wayman, A. K. Jai"FVC2002: Fingerprint verification competition" in Proc. Int. Conf. Pattern Recognition (ICPR), Quebec City,QC, Canada, August 2002, pp. 744-747

[8] A. Ross, A. K. Jain, "Information fusion in biometrics", Pattern Recognition Letters 24 (2003) 2115-2125, available at http://www.computerscienceweb.com/.

[9] K.Delac,M.Grgic “A Survey of Biometric Recognition Methods" $46^{\text {th }}$ international symposium Electronics in Marine,Elmar-2004,16-18june 2004

[10] Rahib Hidayat abiyev and kemal ihsan kilic "Robust Feature Extraction and iris recognition for Biometrics personal identification" (2011).

[11] V.Roselin.E.C,L.M.Waghmare "Iris Biometrics Recognition for Person Identification in Security Systems "International Journal of Computer Application (0975-8887) Volume 24No.9,june 2011.

[12] Makram Nabti,Ahmed Bouridane "An effective and fast iris recognition system based on a combined multiscale feature extraction technique "accepted 26 June 2007.

[13] "individual Biometrics: Iris Scan" http://ctl.ncsc.dni.us/biomet\%20web/BMIris.html

[14] Iridian Technologies, “ Historical Timeline” http://www.iridiantech.com/about.php?page $=4$

[15] Kelly Smith, “Iris Patent Question,” 9 June 2005

[16] Debnath Bhattacharyya,samir kumar.b “ Iris Texture Analysis and Feature Extraction for Biometric pattern Recognition”.

[17] Techshino "review on 10 june 2013" http://en.techshino.com/tech/detail.html?id=3.

[18] Zhaofeng He, Tieniu Tan, Zhenan Sun and Xianchao Qiu, "Robust eyelid eyelash and shadow localization for iris recognition", 15th IEEE International Conference on image processing (ICIP) 12-15 Oct, pp. 265-268, 2008

[19] Amrita,Jaspreet SighCheema, " Detection and removal of Noises in Iris Recognition System-A Review” April-2013. 